\section{OP28 HOW DO DUTCH PRIMARY CARE PROVIDERS OVERCOME BARRIERS TO ADVANCE CARE PLANNING WITH OLDER PEOPLE? A QUALITATIVE STUDY}

IJ Glaudemans*, 'A de Jong, 'J Wind, ${ }^{2}$ B Onwuteaka Philipsen, 'D Willems. ${ }^{1}$ Amsterdam UMC, Amsterdam Public Health research institute, location AMC Amsterdam, Netherlands; ${ }^{2}$ Amsterdam UMC location VUmc, Amsterdam, Netherlands

\subsection{6/spcare-2019-ACPICONGRESSABS.28}

Background Few older people benefit from advance care planning (ACP), due to several barriers related to primary care professionals, such as insufficient knowledge, negative beliefs and a lack of time. Information on overcoming these barriers is limited. We assumed primary care professionals experienced in ACP with older patients are likely to have learned how to overcome these barriers. Therefore we investigated how primary care professionals, experienced in ACP with older patients, overcome these barriers.

Methods A qualitative study, based on semi-structured interviews, among a purposive sample of 14 Dutch primary care professionals experienced in ACP with older people. Transcripts were thematically analysed.

Results We interviewed eight general practitioners (GPs), three nurses and three elderly care physicians, experienced in ACP with older people. Respondents overcame their own insufficient knowledge and skills, as well as their negative attitudes and beliefs by gaining experience through practicing ACP in their daily practices, exchanging and reflecting on those experiences with peers, pursuing continuing education, teaching and participating in research. To overcome patients' and families' lack of initiative and openness to ACP, respondents prepared them for further steps in ACP. To overcome a lack of time, respondents used tools and information communication technology, delegated parts of ACP to other primary care professionals, acquired financing and systematized documentation of ACP.

Conclusions Primary care professionals can overcome barriers to ACP with older patients by practicing, reflecting on experiences and pursuing continuing education, by preparing patients and involving family and by investing in support to approach ACP more efficiently.

\section{OP29 'WHILE MY THINKING IS CLEAR': OUTCOMES FROM A FEASIBILITY PILOT OF A MULTIDISCIPLINARY, STEP-WISE PATHWAY FOR ACP IN FAMILY MEDICINE}

A Tan*, C Paterson, R Bernacki, R Urquhart, D Barwich, M Howard. Cumming School of Medicine, University of Calgary, Calgary, Canada

\subsection{6/spcare-2019-ACPICONGRESSABS.29}

Background Advance care planning (ACP) and goals of care (GCD) discussions with patients align with the tenets of patient-centred shared decision-making central to family medicine (FM). We sought to determine whether a multidisciplinary pathway is feasible in family medicine to enable effective ACP conversations. This pathway reorders Ariadne Lab's Serious Illness Conversation Guide (SICG) with a values clarification tool in a step-wise approach to ACP.

Methods Mixed-methods feasibility pilot study of pathway implementation in an urban FM clinic in Alberta, Canada. We recruited community-dwelling patients age 60 or older with indications of frailty (multi-morbidity, unplanned hospitalizations), and their surrogate decision-maker (SDM). An allied health professional initiated the ACP pathway, which preceded an appointment with the family physician (FP) to complete the SICG discussion. We conducted a survey of patients and SDMs, and a focus group with clinicians to evaluate feasibility, acceptability and perceived impact.

Results Nine patients, seven SDMs, and four clinicians participated in the pilot. All patients and SDMs rated the process as "very good" or "excellent". Eight patients and two SDMs reflected that discussing and documenting their preferences helped them feel more prepared for future illness, and that involving SDMs was essential. Clinicians found the pathway and SICG improved their skills and empowered them to facilitate these conversations more effectively.

Conclusions This pathway that adapts use of the SICG was acceptable and effective for all participants. The pathway fits well into FM as the trusting relationship between the patient and FP provides the foundation for these meaningful conversations.

\section{OP30 SCOPING REVIEW ABOUT WEB-BASED ADVANCE CARE PLANNING PROGRAMS}

${ }^{1} \mathrm{D}$ van der Smissen*, ${ }^{1} \mathrm{~A}$ Overbeek, ${ }^{2} \mathrm{~S}$ Van Dulmen, ${ }^{3} \mathrm{~J}$ Van Gemert-Pijnen, ${ }^{1} \mathrm{~A}$ van der Heide, ${ }^{1} \mathrm{~J}$ Rietjens, ${ }^{1} \mathrm{I}$ Korfage. 'Erasmus University Medical Center, Rotterdam, Netherlands; ${ }^{2}$ NIVEL, Utrecht, Netherlands; ${ }^{3}$ University of Twente, Enschede, Netherlands

\subsection{6/spcare-2019-ACPICONGRESSABS.30}

Background Advance Care Planning (ACP) enables persons to define goals and preferences for future medical treatments and care, to discuss, record and review these. Potentially, web-based programs can support patients in ACP. However, an overview of their effectiveness and feasibility is lacking. Methods To provide an overview of the content, feasibility and effectiveness of web-based, interactive and patient-centered ACP programs, we systematically searched in 7 databases. We extracted data using the EAPC consensus concept of ACP as our framework.

Results The search identified 3434 records; 21 studies were included. Three additional studies were identified by hand search. The 24 studies evaluated 11 web-based ACP programs, developed in the USA (10) and Ireland (1). Most programs addressed exploration of goals, values and preferences, and ACP communication. Users considered programs as easy to use (7/7 studies) and not burdensome (7/8 studies). Users were satisfied with the programs (10/10 studies). ACP communication (11/11 studies) and ACP documentation (14/16 studies) increased. Two studies evaluated concordance between preferred and provided care. Designs of 10 studies allowed comparison before/after completing ACP programs or between study groups.

Conclusion(s) Most web-based ACP programs contain the important elements of ACP. Studies reported that programs tended to be effective and feasible. Evaluations of concordance between preferred and provided care are scarce. Web-based programs have potential to support patients, and scale up ACP. However, since many studies did not assess differences before/after or between groups, outcomes should be interpreted with caution. 